\title{
Integración de tecnologías de apoyo para educación especial
}

\author{
Miguel Ángel Guillomía, Jorge Falcó Boudet, José Ignacio Artigas \\ Grupo de Electrónica de Potencia y Microelectrónica (GEPM) \\ Instituto de Investigación en Ingeniería de Aragón (I3A) \\ Universidad de Zaragoza, Mariano Esquillor s/n, 50018, Zaragoza, Spain. \\ Tel. +34-976762707, e-mail: 601084@unizar.es
}

\section{Resumen}

Se presenta un resumen de la tesis cuyo objetivo es realizar una plataforma de servicios de AAL como tecnología de apoyo para educación especial, enfocada en adiestrar y dar apoyo tanto a la capacidad cognitiva del alumno como a la emocional y social.

\section{Motivación e introducción}

La idea inicial surgió del trabajo previo con personal del CPEE Alborada de Zaragoza. Se trata de aprovechar los conocimientos y habilidades adquiridas por los alumnos en aprendizajes anteriores al manejo de los programas que se les ofrecen. En concreto la utilización habitual de sistemas de comunicación aumentativa y alternativa (CAA), como el proyecto TICO de AraSuite [1], ha logrado el aprendizaje del significado de pictogramas y sus secuencias para expresar ideas e intenciones, consiguiendo en los alumnos un instrumento de acceso cognitivo potente, modular, adaptable a las capacidades individuales y con un esquema o gramática trasladable al manejo de otros constructos cognitivos, como puede ser el manejo de un control de entorno (CE) o la comprensión del paso del tiempo.

Se trabajará en una herramienta para servicios heterogéneos en un sistema de TIC simple, modular, ampliable y abierto, para alcanzar una mayor accesibilidad cognitiva y para explorar lo lejos que los niños pueden llegar en la utilización del CE y servicios similares con la capacitación escolar adecuada. Se busca que esos servicios mejoren la autonomía de los niños.

\section{Objetivos}

El primer objetivo es desarrollar una plataforma de bajo coste que incluya un $\mathrm{CE}$ con interfaz de usuario análoga a TICO, el sistema de CAA que se utiliza en el colegio.
El segundo es adaptar la plataforma para ofrecer al profesor un instrumento para el adiestramiento del alumno en funciones cognitivas básicas y funciones de acceso e interacción básicos.

El tercero es adaptar la tecnología de apoyo de OT existente a las necesidades del adiestramiento y apoyo en OT del colegio Alborada y evaluar el beneficio de la intervención en OT en la población elegida.

El cuarto es incluir un servicio de contención conductual como paso previo al adiestramiento en gestión emocional.

El quinto es integrar en el aula un servicio de anticipación de contextos como apoyo al adiestramiento en auto-conocimiento del estado emocional del alumno y su potencial regulación emocional en situaciones de interacción social y otras

\section{Metodología}

La Figura 1 muestra las etapas seguidas en el desarrollo de la tesis. La plataforma tecnológica se basa en una computadora de placa Rapsberry PI y diversos interfaces y dispositivos comerciales de CE. Para la evaluación de las mejoras en los alumnos se ha utilizado la metodología de estudio de casos.

\section{Conclusiones}

Una vez adaptada la tecnología se probó el grado de aprendizaje y el alcance del beneficio por usar estos sistemas integrados con su lenguaje de CAA. Se concluyó que el uso de interfaces análogos a los de CAA capacitaba el aprendizaje de este servicio y plataforma, y que el uso de sistemas de CE en población de educación especial mejora su grado de autonomía personal.

Se desarrolló una plataforma que integra un sistema de servicios capaz de ofrecer al alumno un

Revista "Jornada de Jóvenes Investigadores del I3A", vol. 8 (Actas de la IX Jornada de Jóvenes Investigadores del I3A -11 de diciembre de 2020). ISSN 2341-4790. 
instrumento para su adiestramiento, que incluye la interfaz adaptable y análoga a CAA utilizado con elementos virtuales que representan elementos reales y asociando acciones sobre ellos. Se comprueba, por observación directa de los profesores, que la combinación de los servicios de CE y CAA ofrece un nuevo método de entrenamiento de funciones básicas: en la comprensión de la función causa-efecto y la relación del ítem abstracto de la interfaz de usuario con la realidad que representa y las acciones a realizar sobre ellos [1].

Se logró adaptar el servicio de OT a las nuevas necesidades del adiestramiento y apoyo en OT. Se modificó el hardware para obtener una tecnología más autónoma, potente y versátil. Se realizó una adaptación del software de interfaz de usuario a su uso mediante paneles similares a TICO, según el criterio de los profesores. Se virtualizó el dispositivo físico de OT, para disponer de él mediante navegador web para alumnos que pudieran tratar con ese nivel de abstracción [3, 4]. El estudio de casos mostró mejora en algunos ítems de la Clasificación internacional del funcionamiento, de la discapacidad y de la salud: CIF [5] de la OMS relacionados con la actividad y con la autonomía personal del alumno.

Se incorporaron herramientas de habilitación cognitiva y de apoyo permitiendo trasladar patrones de aprendizajes previos. Se consiguió adaptar una herramienta de apoyo cognitivo en una plataforma de servicios, centrada en el uso del estilo de una interfaz conocida. También se ha podido concluir la eficacia de la realimentación del paso del tiempo en la contención conductual de forma subjetiva por parte del profesorado, dejando la formalización de esta evaluación para acciones futuras.

Se consiguió integrar dispositivos de estimulación multisensorial (especialmente elementos de escenografía con interfaz DMX) y de regulación emocional en el aula y trabajar con ellos, demostrando que son un apoyo al adiestramiento según el estado emocional del alumno y su potencial regulación emocional.

Debido a los cambios inesperados del curso 20192020 debido a la COVID-19, ha quedado pendiente la evaluación de la mejora en la regulación emocional para la autonomía del alumno en sus facetas emocional y social.

\section{REFERENCIAS}

[1]. «AraSuite», 2020. [En línea]. Disponible en: https://sourceforge.net/projects/arasuite/. [Accedido: 12-nov-2020.

[2]. GUILLOMÍA, M.A., FALCÓ BOUDET, JORGE, ARTIGAS, JI,.AGUSTÍN, A.S. "AAL Platform with a "de facto" Standard Communication Interface (TICO): Training in Home Control in Special Education», Sensors (Switzerland), vol. 17, n.o 10, oct. 2017.

[3]. GUILLOMÍA, M.A., FALCÓ BOUDET, JORGE, ARTIGAS, JI,. Time Orientation Training in AAL. 9TH International Symposium on Ambient Intelligente, Toledo (Spain), 2018.

[4]. GUILLOMÍA, M.A., FALCÓ BOUDET, JORGE, ARTIGAS, JI,. «Time Orientation Technologies in Special Education». Sensors (Switzerland), vol. 19, n.o 11, jun. 2019

[5]. ORGANIZACIÓN MUNDIAL DE LA SALUD, «Clasificación internacional del funcionamiento, de la discapacidad y de la salud: CIF.», 2001.

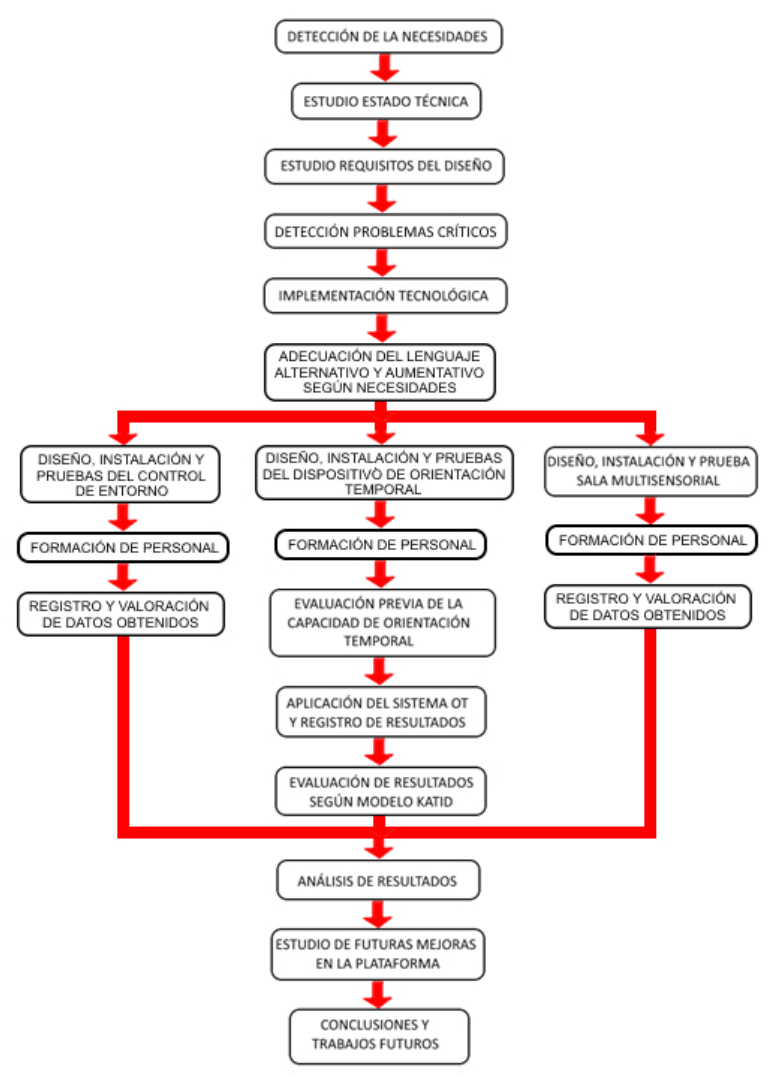

Figura 1. Etapas de la investigación de la tesis 